\title{
Electroosmotic flow of a power-law fluid in a slit microchannel with gradually varying channel height and wall potential
}

\author{
Cheng Qi, Chiu-On $\mathrm{Ng}^{1}$ \\ Department of Mechanical Engineering, The University of Hong Kong, \\ Pokfulam Road, Hong Kong
}

April 20, 2015

\begin{abstract}
A mathematical model based on the lubrication theory is presented for quasi-onedimensional electroosmotic flow of a power-law fluid through a slit microchannel with undulating and non-uniformly charged walls. The channel height and the wall potential may vary periodically with axial position, with a wavelength much longer than the average channel height. Owing to the nonlinear rheology, the pressure gradient that is internally induced to satisfy continuity of flow has to be found numerically. A trial-anderror method is adopted to search for a flow rate that will give rise to an axial pressure gradient distribution with a zero average over one wavelength of the channel. When the flow behavior index is equal to the reciprocal of an integer, polynomial equations relating the flow rate and the local pressure gradient can be deduced, which will greatly facilitate the seeking of the solution by trial and error. Numerical results are also presented to illustrate how the flow behavior index may qualitatively change the combined effect of the geometric and electrokinetic wall patterns on the flow rate.
\end{abstract}

Keywords: electroosmotic flow; power-law fluid; lubrication approximation.

\footnotetext{
${ }^{1}$ Corresponding author. Tel: (852) 2859 2622; Fax: (852) 28585415; E-mail address: cong@hku.hk (C.-O. $\mathrm{Ng})$.
} 


\section{Introduction}

In microfluidics, a fluid is often transported in a microchannel by means of an applied electric field. Such electrically driven flow, which draws upon the unbalanced charge distribution in the electric double layer (EDL) formed near a charged surface, is commonly called the electroosmotic (EO) flow. Since the pioneering work by Burgreen and Nakache [1] five decades ago, EO flow in microchannels has been extensively studied, especially in the past twenty years. These existing studies, whether theoretical or experimental, are mostly for EO flow of Newtonian fluids. Effects of non-Newtonian behaviors on EO flow have not received much attention until recently. The need for an in-depth understanding of non-Newtonian EO flow stems from the fact that microfluidic applications often involve complex fluids such as polymeric solutions and bio-fluids [2].

Das and Chakraborty [3] and Chakraborty [4] were among the first who presented theoretical models for EO flow of non-Newtonian fluids in microchannels. These authors adopted the power-law model to describe the non-Newtonian rheology. EO flows of other non-Newtonian fluids, such as Bingham [5], viscoelastic [6], and viscoplastic [7,8] materials, have also been investigated. To this date, the most chosen rheological model for non-Newtonian EO flow has been the power-law model; some typical works, among many others, are found in Refs. [9-15]. The power-law model, also known as the Ostwald-de Waele model, is a relatively simple twoparameter model, by which the shear-thinning, Newtonian, or shear-thickening behaviors can be conveniently represented by the flow behavior index being less than, equal to, or larger than unity, respectively.

Some noticeable analytical solutions for EO flow of power-law fluids in microchannels have been obtained by Yang and his collaborators [9, 11, 16, 17]. One remarkable finding by these authors is an expression for the generalized Smoluchowski slip velocity for power-law fluids. For EO flow under the Debye-Hückel approximation (i.e., very small electric potentials), they also found closed-form analytical solutions for some particular values of the flow behavior index $n$ (namely, $n=1,1 / 2$ and 1/3), and approximate analytical solutions for an arbitrary value of $n$. These and other analytical studies on EO flow of non-Newtonian fluids are, however, limited to uniform channels of simple geometry (e.g., a uniform parallel-plate or 
circular channel) such that the flow is steady and unidirectional, with the velocity depending on one transverse coordinate only. This is because, for such steady one-dimensional flow, the shear stress distribution can be determined a priori, which will then allow the velocity to be found straightforwardly by integration. For a flow behavior index $n$, the integrand has the form of a certain function raised to the exponent of $1 / n$. If the function is an elementary function and $n$ is the inverse of an integer, a closed-form analytical expression can be obtained for the velocity profile.

Non-uniformities are known to occur to microchannels. For example, the zeta potential, or the electric potential at the shear plane in the EDL, may vary spatially by construction or owing to unavoidable surface defects during fabrication. For Newtonian fluids, many have studied EO flow in microchannels with non-uniformly charged walls [18-22]. The problem is of practical importance because non-uniform wall charge may lead to secondary flow in the form of flow separation and recirculation. The problem becomes more interesting when non-uniform wall charge interacts with an undulating wall shape, as has been investigated by Ajdari $[23,24]$. He showed that the combined effect of periodic wall charge and shape modulation is to generate net flow even if the walls are on average electro-neutral. Charge modulation alone can only produce periodic convective cells, but will not generate net flow. Net effects may happen only when the symmetry of forward-backward flow induced by equal positive-negative charge distributions is broken by the superposition of a wavy wall. The net flow can be in a direction as if it were uniformly negatively charged even when the average wall charge is positive, and vice versa. Ghosal [25] also studied EO flow in channels where the cross-section and surface charge may vary slowly in the axial direction. These existing models are, however, for Newtonian fluids only. The desire for extending the work to non-Newtonian fluids has motivated the present study.

In this paper, we aim to study EO flow of a power-law fluid in a non-uniform slit microchannel with periodic axial variations of wall charge and channel height. The objective is to develop a model that enables us to examine the effects due to the power-law rheology on the interaction between the two wall patterns (one electrokinetic and one geometric) in controlling the flow through the channel. The flow is intrinsically two dimensional, and hence the analytical approach mentioned above will no longer be applicable. We shall neverthe- 
less simplify the present model by means of the lubrication approximation, by which the problem can be formulated in a quasi-one-dimensional manner, thereby avoiding solving the momentum equations in full. The challenging part in the present problem is to determine an unknown pressure distribution along the channel. The pressure as a function of the axial coordinate, which is internally induced so as to maintain a constant flow rate through a channel with axial non-uniformities, has to be found numerically owing to the nonlinear interaction between the hydrodynamic and electric forcings for a non-Newtonian fluid. This distinguishes the present study from previous studies by the authors [26,27], which also look into EO flow of power-law fluid in a non-uniform channel, but are simplified by the use of the Newtonian Helmholtz-Smoluchowski slip boundary condition on taking into account a near-wall Newtonian depletion layer.

Our problem is defined in further detail in Sec. 2, where a mathematical formulation based on the lubrication approximation and the Debye-Hückel approximation is presented. Highly nonlinear equations are to be solved for the flow rate, which is a constant, and the pressure gradient distribution, which is a periodic function of the axial coordinate. To this end, a trialand-error solution method is used, as described in Sec. 3. This method involves the searching for a flow rate that leads to a pressure distribution where the net pressure change over one wavelength is zero. For particular values of the flow behavior index $n$, namely equal to the reciprocal of an integer, polynomial equations can be derived to relate the flow rate and the local pressure gradient. When the integer is an even integer, the type of stress distribution has to be identified on deriving these polynomial equations. The availability of these analytical relationships will alleviate the numerical efforts involved in finding the solution. In Sec. 4, some physical discussion is presented. We shall look into how the flow behavior index may modify the combined effect of the wall undulation and the charge modulation on the EO flow. We shall show that the dependence of the flow rate on the wall pattern parameters may change with the flow behavior index, not only quantitatively, but also qualitatively. 


\section{Mathematical formulation}

Our problem is to consider steady electroosmotic (EO) flow of a power-law fluid through a slit microchannel, of which the channel height as well as the wall potential may vary gradually and periodically in the streamwise direction. Figure 1 shows a definition sketch of the problem, where $(x, y)$ are the axial and transverse coordinates, and the $x$-axis is along the centerline of the channel. For simplicity, only flow that is symmetrical about the $x$-axis is considered: the upper/lower walls are at $y= \pm h(x)$ and the wall potential at either wall is given by $\zeta=\zeta(x)$, both being periodic functions of $x$ with the same wavelength $L$. The wavelength $L$, which is the length scale for variations of velocity in the axial direction, is assumed to be much longer than the mean channel height: $L \gg h_{0}$. With this sharp contrast in length scales, we further assume that the Reynolds number of the flow is so small that the lubrication approximation $[28,29]$ can be applied here. The flow is therefore nearly one dimensional: the axial velocity $u$ is an order of magnitude larger than the transverse velocity $v$. Also, the inertia of the flow can be ignored, and the change of $u$ in the $x$-direction is much milder than that in the $y$-direction.

In this work, the near-wall Newtonian depletion layer is assumed to be so thin that it is completely covered by the EDL, and therefore its effect on the bulk flow can be ignored. This will be valid when the thickness of the depletion layer, which is approximately the radius of gyration of the molecules making up the nonlinear rheology, is of the order of nanometers [30], while the thickness of the EDL, which depends on the bulk ion concentration, is of the order of hundreds of nanometers. On ignoring the depletion layer, the fluid is taken to be homogeneously non-Newtonian throughout the flow domain. The rheological behavior exhibited by a power-law fluid under simple shear is as follows:

$$
\tau=\mu\left|\frac{\mathrm{d} u}{\mathrm{~d} y}\right|^{n-1} \frac{\mathrm{d} u}{\mathrm{~d} y}=\left\{\begin{array}{ll}
\mu\left(\frac{\mathrm{d} u}{\mathrm{~d} y}\right)^{n} & \text { for } \frac{\mathrm{d} u}{\mathrm{~d} y}>0 \\
-\mu\left(-\frac{\mathrm{d} u}{\mathrm{~d} y}\right)^{n} & \text { for } \frac{\mathrm{d} u}{\mathrm{~d} y}<0
\end{array},\right.
$$

where $\tau$ is the shear stress, $\mu$ is the flow consistency, and $n$ is the power-law or flow behavior index of the fluid. The shear-thinning, Newtonian, and shear-thickening behaviors are exhibited when $n<1,=1,>1$, respectively. On rearranging the terms, the equation above can 
be written as

$$
\frac{\mathrm{d} u}{\mathrm{~d} y}=\mu^{-\frac{1}{n}}|\tau|^{\frac{1}{n}-1} \tau= \begin{cases}\left(\frac{\tau}{\mu}\right)^{\frac{1}{n}} & \text { for } \tau>0 \\ -\left(-\frac{\tau}{\mu}\right)^{\frac{1}{n}} & \text { for } \tau<0\end{cases}
$$

Ignoring gravity, the Cauchy momentum equation for the fluid flow under the lubrication approximation $[26]$ is

$$
P_{x}+\frac{\partial \tau}{\partial y}+\rho_{e} E_{x}=0
$$

where $P_{x}=-\mathrm{d} p / \mathrm{d} x$ is the pressure gradient, $\rho_{e}(y)$ is the free charge density in the electric double layer (EDL) of the channel wall, and $E_{x}$ is the applied axial electric field. Here, $E_{x}=$ constant is an externally applied electric field, while $P_{x}=P_{x}(x)$ is an internally induced pressure gradient. The pressure gradient, as a hydrodynamic forcing, is triggered by the need to maintain a constant mass flux through the channel with axially varying channel height and wall potential distribution. By symmetry about the centerline $(y=0)$, it suffices for us to consider, from here onward, flow in the upper half of the channel: $0 \leq y \leq h(x)$.

The electric potential $\psi(y)$, measured relative to the bulk solution phase, where $\psi$ is zero, is related to the charge density $\rho_{e}$ by the Poisson equation:

$$
\frac{\mathrm{d}^{2} \psi}{\mathrm{d} y^{2}}=-\frac{\rho_{e}}{\epsilon}
$$

where $\epsilon$ is the dielectric permittivity of the liquid electrolyte.

With Eq. (4), Eq. (3) can be integrated to give the stress distribution

$$
\tau=-P_{x} y+\epsilon E_{x} \frac{\mathrm{d} \psi}{\mathrm{d} y}
$$

where the symmetry conditions, $\tau=0$ and $\mathrm{d} \psi / \mathrm{d} y=0$ at $y=0$, have been used.

For the flow considered in our problem, the ionic flux $j_{i}$ of every species due to electrical forces, fluid convection and Brownian diffusion in the $y$-direction is given by the NernstPlanck relationship [31] as follows:

$$
j_{i}=-D_{i} \frac{\mathrm{d} n_{i}}{\mathrm{~d} y}-\frac{z_{i} e n_{i} D_{i}}{k_{B} T} \frac{\mathrm{d} \psi}{\mathrm{d} y}
$$

where $D_{i}$ is the diffusion coefficient, $n_{i}$ is the ion distribution, $z$ is the valency, $e$ is the fundamental charge, $k_{B}$ is Boltzmann's constant, and $T$ is the absolute temperature. The 
equation obtained above neglects convective transport (based on the assumption of a very small ionic Péclet number) as there is virtually no flow in the transverse direction. As channel walls are impermeable, there is no ionic flux such that $j_{i} \equiv 0$, which gives the Boltzmann distribution:

$$
n_{i}(y)=n_{i, \infty} \exp \left(-\frac{z_{i} e \psi}{k_{B} T}\right),
$$

where $n_{i, \infty}$ is the bulk concentration for the species $i$. Considering a symmetric $(z: z)$ electrolyte solution for simplicity, it holds that $z_{+}=-z_{-}=z$ and $n_{+\infty}=n_{-\infty}=n_{\infty}$. Given that $\rho_{e}=e \sum_{i} z_{i} n_{i}$, the electric charge density can be further written as

$$
\rho_{e}=-2 z e n_{\infty} \sinh \left(\frac{z e \psi}{k_{B} T}\right) .
$$

Putting Eq. (8) into Eq. (4) will form the well-known Poisson-Boltzmann equation. On further assuming small potentials, ze $\psi / k_{B} T \ll 1$, the Poisson-Boltzmann equation can be linearized to

$$
\frac{\mathrm{d}^{2} \psi}{\mathrm{d} y^{2}}=\kappa^{2} \psi,
$$

where $\kappa=\left(2 z^{2} e^{2} n_{\infty} / \epsilon k_{B} T\right)^{1 / 2}$ is termed the Debye parameter, the inverse of which is the Debye shielding length of the EDL. The linearization of the Poisson-Boltzmann equation is also known as the Debye-Hückel approximation.

Under the assumption that the EDL is much thinner than the channel height, i.e., $\kappa h \gg 1$, the boundary conditions for Eq. (9) can be stated as follows:

$$
\begin{array}{cl}
\psi=\zeta & \text { at } \bar{y}=0, \\
\frac{\mathrm{d} \psi}{\mathrm{d} \bar{y}}=0 & \text { at } \bar{y} \gg 1,
\end{array}
$$

where $\bar{y}=\kappa(h-y)$ is a stretched coordinate, and $\zeta=\zeta(x)$ is the wall zeta potential. Equations (9)-(11) have the following solution for the potential distribution:

$$
\psi(\bar{y})=\zeta e^{-\bar{y}} \quad \text { or } \quad \psi(y)=\zeta e^{-\kappa(h-y)}
$$

Owing to the exponential decay, the electric potential and its gradient are essentially zero in the core of the channel. Substituting the expression above for $\psi$ in Eq. (5), we now obtain the stress distribution as

$$
\tau=-P_{x} y+\epsilon \kappa E_{x} \zeta e^{-\kappa(h-y)} \quad \text { in } 0 \leq y \leq h(x),
$$


To facilitate discussion, let us introduce the following normalized variables (distinguished by an overhead caret):

$$
\left.\begin{array}{c}
\hat{x}=x / L, \quad(\hat{y}, \hat{h})=(y, h) / h_{0}, \quad \hat{u}=u / u_{0}, \\
\hat{\kappa}=\kappa h_{0}, \quad \hat{\zeta}=\zeta / \zeta_{0}, \quad\left(\hat{\tau}, \hat{P}_{x}\right)=\left(\tau, P_{x} h_{0}\right) / \tau_{0},
\end{array}\right\}
$$

where $h_{0}$ is half the average height of the channel, $\zeta_{0}$ is a characteristic value of the wall potential, and $u_{0}$ and $\tau_{0}$ are scalings for the axial velocity and shear stress given by

$$
\begin{gathered}
u_{0}=\frac{n}{\kappa}\left(\frac{\tau_{0}}{\mu}\right)^{\frac{1}{n}}, \\
\tau_{0}=-\epsilon \kappa \zeta_{0} E_{x}>0 .
\end{gathered}
$$

To generate flow in the positive $x$-direction, $\zeta_{0}$ and $E_{x}$ must be of opposite signs, and hence $\tau_{0}$ has to be a positive quantity. One may recognize that $u_{0}$ is the generalized Smoluchowski velocity for a power-law fluid, as was first derived by Zhao et al. [9].

In terms of the normalized variables, the stress distribution given in Eq. (13) becomes

$$
\hat{\tau}=-\hat{P}_{x} \hat{y}-\hat{\zeta} e^{-\hat{\kappa}(\hat{h}-\hat{y})} \quad \text { in } 0 \leq \hat{y} \leq \hat{h}(\hat{x}),
$$

while the power-law constitutive equation given in Eq. (2) becomes

$$
\frac{\mathrm{d} \hat{u}}{\mathrm{~d} \hat{y}}=\frac{\hat{\kappa}}{n}|\hat{\tau}|^{\frac{1}{n}-1} \hat{\tau}
$$

The integration of the equation above, on using the no-slip condition at the wall $\hat{u}(\hat{y}=\hat{h})=0$, gives the velocity

$$
\hat{u}=-\frac{\hat{\kappa}}{n} \int_{\hat{y}}^{\hat{h}}|\hat{\tau}|^{\frac{1}{n}-1} \hat{\tau} \mathrm{d} \hat{y}
$$

where $\hat{\tau}$ is given by Eq. (17). The non-dimensional volume flow rate through the channel is then given by

$$
\hat{q}=-\frac{\hat{\kappa}}{n} \int_{0}^{\hat{h}} \int_{\hat{y}}^{\hat{h}}|\hat{\tau}|^{\frac{1}{n}-1} \hat{\tau} \mathrm{d} \hat{y} \mathrm{~d} \hat{y}
$$

where $\hat{q}$ is the flow rate normalized by $2 u_{0} h_{0}$. By continuity, $\hat{q}$ is independent of the axial coordinate $\hat{x}$. Another condition is obtainable from the periodicity of the flow. In the absence of applied pressure forcing, the net change in pressure over one wavelength of the channel is zero. Therefore,

$$
\left\langle\hat{P}_{x}\right\rangle \equiv \int_{0}^{1} \hat{P}_{x} \mathrm{~d} \hat{x}=\hat{p}(0)-\hat{p}(1)=0,
$$

where the angle brackets denote axial averaging over one wavelength of the channel. 


\section{Solution methods}

For given $n, \hat{\kappa}, \hat{h}(\hat{x})$ and $\hat{\zeta}(\hat{x})$, the problem is solved when $\hat{q}$ and $\hat{P}_{x}(\hat{x})$ are found such that Eqs. (20) and (21) are satisfied. For a general value of the power-law index $n$, this is a highly nonlinear problem that can be solved numerically by a method of trial-and-error as follows. First, the domain $0 \leq \hat{x} \leq 1$ is subdivided into equal parts by a number of points $\hat{x}_{i}$. Second, with a trial value of $\hat{q}$, the pressure gradient at each point $\hat{P}_{x}\left(\hat{x}_{i}\right)$ is then found from Eq. (20). Third, with the obtained values of $\hat{P}_{x}\left(\hat{x}_{i}\right)$, the pressure gradient over the domain is numerically integrated to determine the error on satisfying Eq. (21). The error will then inform the next trial value of $\hat{q}$. These steps will be repeated until the error, which amounts to a non-zero pressure difference in one wavelength, becomes sufficiently small. Since Eq. (20) is nonlinear and involves a double integral, the finding of $\hat{P}_{x}$ at each point also needs some tedious trial-and-error computations. The searching of a solution satisfying Eqs. (20) and (21) is in general time consuming. It may take a few hours of computer time to generate one solution.

For some particular values of $n$, namely when $n$ equals the reciprocal of an integer, the problem can be solved much more easily. The cases for several such special values of $n$ are discussed below.

\subsection{For $n=1$ (Newtonian)}

The problem can be solved analytically when $n=1$. For this Newtonian limit, the velocity profile is

$$
\hat{u}_{n=1}=\frac{\hat{\kappa} \hat{P}_{x}}{2}\left(\hat{h}^{2}-\hat{y}^{2}\right)+\hat{\zeta}\left[1-e^{-\hat{\kappa}(\hat{h}-\hat{y})}\right]
$$

the integration of which gives the flow rate

$$
\hat{q}_{n=1}=\frac{\kappa \hat{h}^{3}}{3} \hat{P}_{x}+\hat{\zeta}\left(\hat{h}-\frac{1}{\hat{\kappa}}\right)
$$

Rearranging terms, the pressure gradient is

$$
\hat{P}_{x}=\frac{3}{\hat{\kappa} \hat{h}^{3}}\left(\hat{q}_{n=1}-\hat{\zeta} \hat{h}+\hat{\kappa}^{-1} \hat{\zeta}\right) .
$$


On applying Eq. (21), the flow rate is found to be

$$
\hat{q}_{n=1}=\frac{\left\langle\hat{\zeta} \hat{h}^{-2}\right\rangle}{\left\langle\hat{h}^{-3}\right\rangle}-\frac{\left\langle\hat{\zeta} \hat{h}^{-3}\right\rangle}{\hat{\kappa}\left\langle\hat{h}^{-3}\right\rangle},
$$

where the angle brackets denote averaging over one wavelength.

\subsection{For $n=1 / 3,1 / 5, \cdots$}

When $n$ is the reciprocal of an odd integer, $1 / n-1$ becomes an even integer, and hence $|\hat{\tau}|^{1 / n-1} \hat{\tau}=\hat{\tau}^{1 / n}$ irrespective of the sign of $\hat{\tau}$. The integrals in Eq. (20) can then be performed without the need to differentiate between various cases of stress distribution. Using the stress distribution $\hat{\tau}(\hat{y})$ given in Eq. (17), the flow rate as a function of the pressure gradient for $n=1 / 3$ and $n=1 / 5$ are obtainable from Eq. (20) as below:

$$
\begin{aligned}
\hat{q}_{n=1 / 3}= & -3 \hat{\kappa} \int_{0}^{\hat{h}} \int_{\hat{y}}^{\hat{h}} \hat{\tau}^{3} \mathrm{~d} \hat{y} \mathrm{~d} \hat{y} \\
= & \left(\frac{3 \hat{\kappa} \hat{h}^{5}}{5}\right) \hat{P}_{x}^{3}+\frac{9 \hat{\zeta}}{\hat{\kappa}^{3}}\left(\hat{\kappa}^{3} \hat{h}^{3}-3 \hat{\kappa}^{2} \hat{h}^{2}+6 \hat{\kappa} \hat{h}-6\right) \hat{P}_{x}^{2} \\
& +\frac{9 \hat{\zeta}^{2}}{4 \hat{\kappa}^{2}}\left(2 \hat{\kappa}^{2} \hat{h}^{2}-2 \hat{\kappa} \hat{h}+1\right) \hat{P}_{x}+\frac{\hat{\zeta}}{3 \hat{\kappa}}(3 \hat{\kappa} \hat{h}-1),
\end{aligned}
$$

which is a cubic polynomial function of $\hat{P}_{x}$, and

$$
\begin{aligned}
\hat{q}_{n=1 / 5}= & -5 \hat{\kappa} \int_{0}^{\hat{h}} \int_{\hat{y}}^{\hat{h}} \hat{\tau}^{5} \mathrm{~d} \hat{y} \mathrm{~d} \hat{y} \\
= & \left(\frac{5 \hat{\kappa} h^{7}}{7}\right) \hat{P}_{x}^{5}+\frac{25 \hat{\zeta}}{\hat{\kappa}^{5}}\left(\hat{\kappa}^{5} \hat{h}^{5}-5 \hat{\kappa}^{4} \hat{h}^{4}+20 \hat{\kappa}^{3} \hat{h}^{3}-60 \hat{\kappa}^{2} \hat{h}^{2}+120 \hat{\kappa} \hat{h}-120\right) \hat{P}_{x}^{4} \\
& +\frac{25 \hat{\zeta}^{2}}{2 \hat{\kappa}^{4}}\left(2 \hat{\kappa}^{4} \hat{h}^{4}-4 \hat{\kappa}^{3} \hat{h}^{3}+6 \hat{\kappa}^{2} \hat{h}^{2}-6 \hat{\kappa} \hat{h}+3\right) \hat{P}_{x}^{3} \\
& +\frac{50 \hat{\zeta}^{3}}{27 \hat{\kappa}^{3}}\left(9 \hat{\kappa}^{3} \hat{h}^{3}-9 \hat{\kappa}^{2} \hat{h}^{2}+6 \hat{\kappa} \hat{h}-2\right) \hat{P}_{x}^{2} \\
& +\frac{25 \hat{\zeta}^{4}}{32 \hat{\kappa}^{2}}\left(8 \hat{\kappa}^{2} \hat{h}^{2}-4 \hat{\kappa} \hat{h}+1\right) \hat{P}_{x}+\frac{\hat{\zeta}}{5 \hat{\kappa}}(5 \hat{\kappa} \hat{h}-1),
\end{aligned}
$$

which is a quintic polynomial function of $\hat{P}_{x}$. The availability of these analytical relationships will greatly expedite finding the values of the pressure gradient distribution $\hat{P}_{x}\left(\hat{x}_{i}\right)$ for a given $\hat{q}$. As a result, the solution seeking by trial-and-error can be accomplished more quickly. Thanks to the condition $\hat{\kappa} \gg 1$, Eqs. (26) and (27) should admit only one real root, thereby avoiding ambiguity in solving these equations for $\hat{P}_{x}$. 


\subsection{For $n=1 / 2,1 / 4, \cdots$}

When $n$ is the reciprocal of an even integer, $1 / n-1$ is an odd integer, and due regard must be paid to the sign of $\hat{\tau}$ on performing the integrals in Eq. (20). At any cross section $\hat{x}=\hat{x}_{i}$, the shear stress distribution across the channel expressed by Eq. (17) is determined by three parameters: $\hat{P}_{x}\left(\hat{x}_{i}\right), \hat{h}\left(\hat{x}_{i}\right)$ and $\hat{\zeta}\left(\hat{x}_{i}\right)$. Different combinations of these parameters may lead to different shear stress distributions, where the stress can be positive, zero or negative at different positions across the section. As shown in Fig. 2 and detailed in Table 1, six possible cases of local stress distribution $\hat{\tau}(\hat{y})$ as well as the corresponding ranges of the three parameters can be identified. For Cases 1 and 6 , the stress is positive for $\hat{y}>0$, and the flow rate is always negative. The opposite is true for Cases 3 and 4 . In these four cases, the flow rate is zero if and only if both $\hat{P}_{x}$ and $\hat{\zeta}$ are zero. For Cases 2 and 5 , the stress near the wall is opposite in sign to that far from the wall, and the flow rate can be positive or negative. Hence, in these two cases, the flow rate can be zero even when $\hat{P}_{x}$ and $\hat{\zeta}$ are non-zero. The height $\hat{y}=\hat{y}_{0}$ at which the stress is zero is given by

$$
\hat{y}_{0}=\hat{h}+\hat{\kappa}^{-1} \ln \left(-\hat{P}_{x} \hat{y}_{0} / \hat{\zeta}\right)
$$

which can be solved by iteration with an initial value of $\hat{y}_{0}=\hat{h}$.

For $n=1 / 2$, the equations governing $\hat{q}$ as a function of the pressure gradient $\hat{P}_{x}$ for the six cases are obtainable from Eqs. (17) and (20) as follows:

$$
\begin{aligned}
& \hat{q}_{n=1 / 2}^{(1,6)}=-2 \hat{\kappa} \int_{0}^{\hat{h}} \int_{\hat{y}}^{\hat{h}} \hat{\tau}^{2} \mathrm{~d} \hat{y} \mathrm{~d} \hat{y} \\
&=\left(-\frac{\hat{\kappa} \hat{h}^{4}}{2}\right) \hat{P}_{x}^{2}-\frac{4 \hat{\zeta}}{\hat{\kappa}^{2}}\left(\hat{\kappa}^{2} \hat{h}^{2}-2 \hat{\kappa} \hat{h}+2\right) \hat{P}_{x}-\frac{\hat{\zeta}^{2}}{2 \hat{\kappa}}(2 \hat{\kappa} \hat{h}-1) \\
& \text { for Cases } 1 \text { and } 6, \\
& \hat{q}_{n=1 / 2}^{(2)}=-2 \hat{\kappa}\left\{\int_{0}^{\hat{y}_{0}}\left[\int_{\hat{y}}^{\hat{y}_{0}}\left(-\hat{\tau}^{2}\right) \mathrm{d} \hat{y}+\int_{\hat{y}_{0}}^{\hat{h}} \hat{\tau}^{2} \mathrm{~d} \hat{y}\right] \mathrm{d} \hat{y}+\int_{\hat{y}_{0}}^{\hat{h}} \int_{\hat{y}}^{\hat{h}} \hat{\tau}^{2} \mathrm{~d} \hat{y} \mathrm{~d} \hat{y}\right\} \\
&=\left(\hat{k} \hat{y}_{0}^{4}-6 \hat{y}_{0}^{3}+\frac{15 \hat{y}_{0}^{2}}{\hat{\kappa}}-\frac{16 \hat{y}_{0}}{\hat{\kappa}^{2}}-\frac{\hat{\kappa} \hat{h}^{4}}{2}\right) \hat{P}_{x}^{2} \\
&-\frac{4 \hat{\zeta}}{\hat{\kappa}^{2}}\left(\hat{\kappa}^{2} \hat{h}^{2}-2 \hat{\kappa} \hat{h}+2\right) \hat{P}_{x}-\frac{\hat{\zeta}^{2}}{2 \hat{\kappa}}(2 \hat{\kappa} \hat{h}-1) \quad \text { for Case } 2, \\
& \hat{q}_{n=1 / 2}^{(3,4)}= 2 \hat{\kappa} \int_{0}^{\hat{h}} \int_{\hat{y}}^{\hat{h}} \hat{\tau}^{2} \mathrm{~d} \hat{y} \mathrm{~d} \hat{y}
\end{aligned}
$$


Table 1: Six possible cases of local stress distribution $\hat{\tau}(0 \leq \hat{y} \leq \hat{h})$, and the corresponding flow rate $\hat{q}$, as determined by the local values of wall potential $\zeta(\hat{x})$, channel height $\hat{h}(\hat{x})$ and pressure gradient $\hat{P}_{x}(\hat{x})$. See Fig. 2 for these six types of stress profiles.

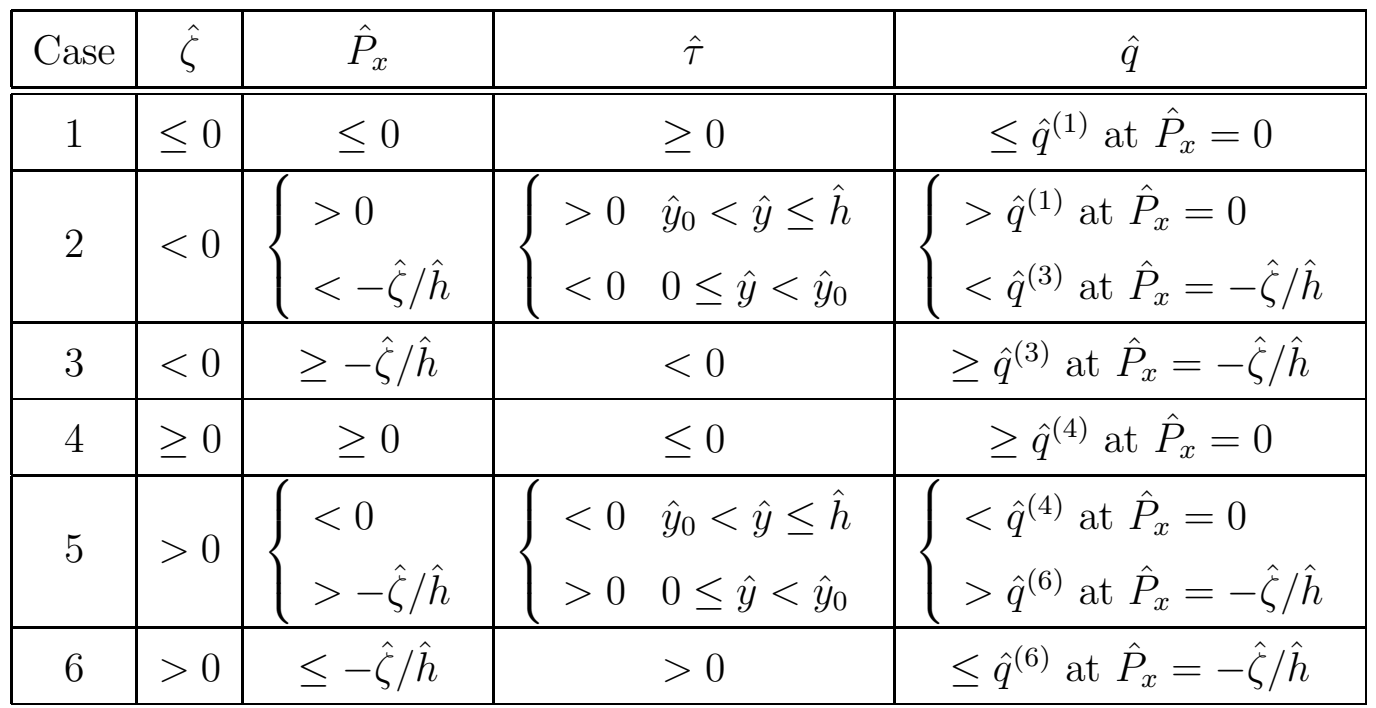

$$
=\left(\frac{\hat{\kappa} \hat{h}^{4}}{2}\right) \hat{P}_{x}^{2}+\frac{4 \hat{\zeta}}{\hat{\kappa}^{2}}\left(\hat{\kappa}^{2} \hat{h}^{2}-2 \hat{\kappa} \hat{h}+2\right) \hat{P}_{x}+\frac{\hat{\zeta}^{2}}{2 \hat{\kappa}}(2 \hat{\kappa} \hat{h}-1)
$$

for Cases 3 and 4,

$$
\begin{aligned}
\hat{q}_{n=1 / 2}^{(5)}= & -2 \hat{\kappa}\left\{\int_{0}^{\hat{y}_{0}}\left[\int_{\hat{y}}^{\hat{y}_{0}} \hat{\tau}^{2} \mathrm{~d} \hat{y}+\int_{\hat{y}_{0}}^{\hat{h}}\left(-\hat{\tau}^{2}\right) \mathrm{d} \hat{y}\right] \mathrm{d} \hat{y}+\int_{\hat{y}_{0}}^{\hat{h}} \hat{y}_{\hat{y}}^{\hat{h}}\left(-\hat{\tau}^{2}\right) \mathrm{d} \hat{y} \mathrm{~d} \hat{y}\right\} \\
= & -\left(\hat{k} \hat{y}_{0}^{4}-6 \hat{y}_{0}^{3}+\frac{15 \hat{y}_{0}^{2}}{\hat{\kappa}}-\frac{16 \hat{y}_{0}}{\hat{\kappa}^{2}}-\frac{\hat{\kappa} \hat{h}^{4}}{2}\right) \hat{P}_{x}^{2} \\
& +\frac{4 \hat{\zeta}}{\hat{\kappa}^{2}}\left(\hat{\kappa}^{2} \hat{h}^{2}-2 \hat{\kappa} \hat{h}+2\right) \hat{P}_{x}+\frac{\hat{\zeta}^{2}}{2 \hat{\kappa}}(2 \hat{\kappa} \hat{h}-1) \quad \text { for Case } 5 .
\end{aligned}
$$

All these are quadratic polynomial functions of $\hat{P}_{x}$.

For $n=1 / 4$, the equations for $\hat{q}$ for the six cases are obtained in a similar manner as follows:

$$
\begin{aligned}
\hat{q}_{n=1 / 4}^{(1,6)}= & -4 \hat{\kappa} \int_{0}^{\hat{h}} \int_{\hat{y}}^{\hat{h}} \hat{\tau}^{4} \mathrm{~d} \hat{y} \mathrm{~d} \hat{y} \\
= & \left(-\frac{2 \hat{\kappa} \hat{h}^{6}}{3}\right) \hat{P}_{x}^{4}-\frac{16 \hat{\zeta}}{\hat{\kappa}^{4}}\left(\hat{\kappa}^{4} \hat{h}^{4}-4 \hat{\kappa}^{3} \hat{h}^{3}+12 \hat{\kappa}^{2} \hat{h}^{2}-24 \hat{\kappa} \hat{h}+24\right) \hat{P}_{x}^{3} \\
& -\frac{3 \hat{\zeta}^{2}}{\hat{\kappa}^{3}}\left(4 \hat{\kappa}^{3} \hat{h}^{3}-6 \hat{\kappa}^{2} \hat{h}^{2}+6 \hat{\kappa} \hat{h}-9\right) \hat{P}_{x}^{2}-\frac{16 \hat{\zeta}^{3}}{27 \hat{\kappa}^{2}}\left(9 \hat{\kappa}^{2} \hat{h}^{2}-6 \hat{\kappa} \hat{h}+2\right) \hat{P}_{x}
\end{aligned}
$$




$$
\begin{aligned}
& -\frac{\hat{\zeta}^{4}}{4 \hat{\kappa}}(4 \hat{\kappa} \hat{h}-1) \text { for Cases } 1 \text { and } 6 \\
& \hat{q}_{n=1 / 4}^{(2)}=-4 \hat{\kappa}\left\{\int_{0}^{\hat{y}_{0}}\left[\int_{\hat{y}}^{\hat{y}_{0}}\left(-\hat{\tau}^{4}\right) \mathrm{d} \hat{y}+\int_{\hat{y}_{0}}^{\hat{h}} \hat{\tau}^{4} \mathrm{~d} \hat{y}\right] \mathrm{d} \hat{y}+\int_{\hat{y}_{0}}^{\hat{h}} \hat{y}_{\hat{y}}^{\hat{h}} \hat{\tau}^{4} \mathrm{~d} \hat{y} \mathrm{~d} \hat{y}\right\} \\
& =\left(\frac{4 \hat{\kappa} \hat{y}_{0}^{6}}{3}-\frac{50 \hat{y}_{0}^{5}}{3}+\frac{1775 \hat{y}_{0}^{4}}{18 \hat{\kappa}}-\frac{9460 \hat{y}_{0}^{3}}{27 \hat{\kappa}^{2}}+\frac{750 \hat{y}_{0}^{2}}{\hat{\kappa}^{3}}-\frac{768 \hat{y}_{0}}{\hat{\kappa}^{4}}-\frac{2 \hat{\kappa} \hat{h}^{6}}{3}\right) \hat{P}_{x}^{4} \\
& -\frac{16 \hat{\zeta}}{\hat{\kappa}^{4}}\left(\hat{\kappa}^{4} \hat{h}^{4}-4 \hat{\kappa}^{3} \hat{h}^{3}+12 \hat{\kappa}^{2} \hat{h}^{2}-24 \hat{\kappa} \hat{h}+24\right) \hat{P}_{x}^{3} \\
& -\frac{3 \hat{\zeta}^{2}}{\hat{\kappa}^{3}}\left(4 \hat{\kappa}^{3} \hat{h}^{3}-6 \hat{\kappa}^{2} \hat{h}^{2}+6 \hat{\kappa} \hat{h}-9\right) \hat{P}_{x}^{2}-\frac{16 \hat{\zeta}^{3}}{27 \hat{\kappa}^{2}}\left(9 \hat{\kappa}^{2} \hat{h}^{2}-6 \hat{\kappa} \hat{h}+2\right) \hat{P}_{x} \\
& -\frac{\hat{\zeta}^{4}}{4 \hat{\kappa}}(4 \hat{\kappa} \hat{h}-1) \quad \text { for Case } 2 \text {, } \\
& \hat{q}_{n=1 / 4}^{(3,4)}=4 \hat{\kappa} \int_{0}^{\hat{h}} \int_{\hat{y}}^{\hat{h}} \hat{\tau}^{4} \mathrm{~d} \hat{y} \mathrm{~d} \hat{y} \\
& =\left(\frac{2 \hat{\kappa} \hat{h}^{6}}{3}\right) \hat{P}_{x}^{4}+\frac{16 \hat{\zeta}}{\hat{\kappa}^{4}}\left(\hat{\kappa}^{4} \hat{h}^{4}-4 \hat{\kappa}^{3} \hat{h}^{3}+12 \hat{\kappa}^{2} \hat{h}^{2}-24 \hat{\kappa} \hat{h}+24\right) \hat{P}_{x}^{3} \\
& +\frac{3 \hat{\zeta}^{2}}{\hat{\kappa}^{3}}\left(4 \hat{\kappa}^{3} \hat{h}^{3}-6 \hat{\kappa}^{2} \hat{h}^{2}+6 \hat{\kappa} \hat{h}-9\right) \hat{P}_{x}^{2}+\frac{16 \hat{\zeta}^{3}}{27 \hat{\kappa}^{2}}\left(9 \hat{\kappa}^{2} \hat{h}^{2}-6 \hat{\kappa} \hat{h}+2\right) \hat{P}_{x} \\
& +\frac{\hat{\zeta}^{4}}{4 \hat{\kappa}}(4 \hat{\kappa} \hat{h}-1) \quad \text { for Cases } 3 \text { and } 4 \text {, } \\
& \hat{q}_{n=1 / 4}^{(5)}=-4 \hat{\kappa}\left\{\int_{0}^{\hat{y}_{0}}\left[\int_{\hat{y}}^{\hat{y}_{0}} \hat{\tau}^{4} \mathrm{~d} \hat{y}+\int_{\hat{y}_{0}}^{\hat{h}}\left(-\hat{\tau}^{4}\right) \mathrm{d} \hat{y}\right] \mathrm{d} \hat{y}+\int_{\hat{y}_{0}}^{\hat{h}} \int_{\hat{y}}^{\hat{h}}\left(-\hat{\tau}^{4}\right) \mathrm{d} \hat{y} \mathrm{~d} \hat{y}\right\} \\
& =\left(-\frac{4 \hat{\kappa} \hat{y}_{0}^{6}}{3}-\frac{50 \hat{y}_{0}^{5}}{3}+\frac{1775 \hat{y}_{0}^{4}}{18 \hat{\kappa}}-\frac{9460 \hat{y}_{0}^{3}}{27 \hat{\kappa}^{2}}+\frac{750 \hat{y}_{0}^{2}}{\hat{\kappa}^{3}}-\frac{768 \hat{y}_{0}}{\hat{\kappa}^{4}}-\frac{2 \hat{\kappa} \hat{h}^{6}}{3}\right) \hat{P}_{x}^{4} \\
& +\frac{16 \hat{\zeta}}{\hat{\kappa}^{4}}\left(\hat{\kappa}^{4} \hat{h}^{4}-4 \hat{\kappa}^{3} \hat{h}^{3}+12 \hat{\kappa}^{2} \hat{h}^{2}-24 \hat{\kappa} \hat{h}+24\right) \hat{P}_{x}^{3} \\
& +\frac{3 \hat{\zeta}^{2}}{\hat{\kappa}^{3}}\left(4 \hat{\kappa}^{3} \hat{h}^{3}-6 \hat{\kappa}^{2} \hat{h}^{2}+6 \hat{\kappa} \hat{h}-9\right) \hat{P}_{x}^{2}+\frac{16 \hat{\zeta}^{3}}{27 \hat{\kappa}^{2}}\left(9 \hat{\kappa}^{2} \hat{h}^{2}-6 \hat{\kappa} \hat{h}+2\right) \hat{P}_{x} \\
& +\frac{\hat{\zeta}^{4}}{4 \hat{\kappa}}(4 \hat{\kappa} \hat{h}-1) \quad \text { for Case } 5 \text {. }
\end{aligned}
$$

All these are quartic polynomial functions of $\hat{P}_{x}$.

As $\hat{\zeta}$ and $\hat{h}$ are functions of $\hat{x}$, the stress distribution may vary among the six possible cases over one wavelength of the channel. In other words, for a fixed flow rate $\hat{q}$, the stress distribution may change with axial position from one type of distribution to another, depending on the local values of $\hat{h}$ and $\hat{\zeta}$. It is necessary, as part of the solution, to determine the stress distribution type before the pressure gradient can be determined at an axial position. 
To this end, use can be made of the ranges of the flow rate given in the last column of Table 1. At each of the discrete points $0 \leq \hat{x}_{i} \leq 1$, one first finds out locally which range of flow rate the trial value of $\hat{q}$ falls into. Then, the corresponding equation is selected to be solved for $\hat{P}_{x}$. The second- or fourth-order polynomial equations have multiple roots, but one should choose the root that is within the range given in the third column of Table 1 . These steps are repeated until a value of $\hat{q}$ is found such that the error of satisfying Eq. (21) is sufficiently small. Our classification of stress distribution described above will greatly expedite the seeking of the solution for $n=1 / 2,1 / 4$.

\section{Discussion}

Let us introduce the following sinusoidal functions for the wall potential and the channel height:

$$
\begin{gathered}
\hat{\zeta}(\hat{x})=\alpha+\beta \cos (2 \pi \hat{x}), \\
\hat{h}(\hat{x})=1+\gamma \cos (2 \pi \hat{x}+\phi),
\end{gathered}
$$

where $\alpha$ is the mean wall potential, $\beta$ and $\gamma$ are the amplitudes of the wall potential and wall shape modulation, respectively, and $\phi$ is a phase shift between the two modulation patterns. These idealized monochromatic waveforms for the geometric and electrokinetic modulation will serve to provide results indicative of the physics involved in the problem. Without loss of generality, only non-negative $\alpha$ and $\beta$ are considered.

In the limiting case of a strictly uniform channel (i.e., flat walls and uniform wall potential distribution), the induced pressure gradient is identically zero $\hat{P}_{x}=0$, and the stress reduces to $\hat{\tau}=-\alpha e^{-\hat{\kappa}(\hat{h}-\hat{y})}$, by which Eq. (20) gives

$$
\hat{q}=\alpha^{1 / n}\left(1-n \hat{\kappa}^{-1}\right) \quad \text { for } \beta=\gamma=0 .
$$

A more general expression for the flow rate in a uniform channel, which is applicable to any $\hat{\kappa}$, has been deduced previously by Zhao et al. [9]. Using the present normalization, their expression reads as follows:

$$
\hat{q}=\alpha^{1 / n} \frac{\left[\left(1-\frac{n}{\hat{\kappa}}\right) e^{\hat{\kappa} / n}+\frac{n-1}{\hat{\kappa}} e^{1 / n}\right]+\frac{2}{\hat{\kappa}(1+2 n)}}{2^{1 / n} \cosh ^{1 / n}(\hat{\kappa})} .
$$


One can check that, for a very thin EDL $(\hat{\kappa} \gg 1)$, the above expression for the flow rate will reduce to ours given in Eq. (39).

Furthermore, with $\hat{\zeta}$ and $\hat{h}$ now specifically given by Eqs. (37) and (38), the flow rate for the Newtonian limit given in Eq. (25) can be evaluated to be

$$
\hat{q}_{n=1}=\frac{2\left(1-\gamma^{2}\right)(\alpha-\beta \gamma \cos \phi)}{2+\gamma^{2}}-\frac{1}{\hat{\kappa}}\left(\alpha-\frac{3 \beta \gamma \cos \phi}{2+\gamma^{2}}\right),
$$

which reduces to the following Newtonian limit of Eq. (39) for a channel of flat walls (i.e., $\gamma=0)$ and any $\beta$ :

$$
\hat{q}_{n=1}=\alpha\left(1-\hat{\kappa}^{-1}\right) \quad \text { for } \gamma=0 .
$$

Obviously, the wall undulation interacts with the wall potential modulation through the term $-\beta \gamma \cos \phi$ in the Newtonian limit $(n=1)$. When either $\gamma$ or $\cos \phi$ is zero, the wall potential modulation $(\beta)$ will have no effect on the flow rate. Whether this statement remains true for non-Newtonian fluid $(n \neq 1)$ is yet to be determined. We shall examine the effect as a function of $n$ arising from the interaction between $\beta$ and $\gamma$ with our numerical discussion below. The computation requires 6 input parameters: $n, \alpha, \beta, \gamma, \phi$ and $\kappa$. Matlab programs were developed to perform the numerical solution seeking by the trial-and-error method described above. Simpson's formula is chosen to evaluate the definite integrals in Eqs. (20) and (21). In all the numerical examples presented below, the following specific parameter values are considered: $n=1 / 5,1 / 2,1,2$, and $\hat{\kappa}=100$.

Let us first look into how the flow rate may be affected by the phase shift $\phi$, as is shown in Fig. 3. Even when the walls are on average electro-neutral (i.e., $\alpha=0$, or zero mean wall potential), a non-zero net flow, positive or negative, can be generated by varying the phase shift between the wall shape and wall potential patterns. Figure 3(a) shows that the flow rate is negative, zero, and positive for $0 \leq \phi<\pi / 2, \phi=\pi / 2$, and $\pi / 2<\phi \leq \pi$, respectively. Such dependence on $\phi$ holds for all values of $n$. The flow rate is the maximum negative when $\phi=0$, and is the maximum positive when $\phi=\pi$. These phases correspond to the cases where the maximum negative/positive wall potential is at the narrowest section of the channel. This correlation between $\hat{q}$ and $\phi$ accords with the well-known fact that, for flow through an axially non-uniform channel, the flow is rate-limited by the conditions prevailing at the narrowest part of the channel. An analytical evidence can be sought by referring to 
the Newtonian case. From Eq. (41),

$$
\hat{q}_{n=1}=-\frac{\beta \gamma \cos \phi}{2+\gamma^{2}}\left[2\left(1-\gamma^{2}\right)-\frac{3}{\hat{\kappa}}\right] \quad \text { for } \alpha=0,
$$

which shows that, for $n=1$, the flow rate is the maximum negative, zero, and maximum positive at $\phi=0, \pi / 2, \pi$, respectively, when $\alpha=0, \beta>0, \gamma>0$. Our numerical results suggest that this statement remains true for $n \neq 1$. One can say that, under the condition $\alpha=0, \hat{q}$ is an odd function of $\phi-\pi / 2$ for any $n$.

The effect of the phase shift on the flow can be very different when $\alpha \neq 0$. Figure 3(b) shows the case where $\alpha=1$. In this case, there is a net flow even when the wall potential is zero at the narrowest section of the channel, which happens when $\phi=0$. Except for $n=1$, the flow rate now varies with the phase shift such that $\hat{q}-\left.\hat{q}\right|_{\phi=\pi / 2}$ is no longer an odd function of $\phi-\pi / 2$. It is remarkable that the flow rate can increase much more dramatically as the phase shift increases toward $\pi$ for smaller $n$. The ratio of $\left.\hat{q}\right|_{\phi=\pi}$ to $\left.\hat{q}\right|_{\phi=0}$ is equal to $2.32,2.98$, $5.39,34.7$ for $n=2,1,0.5,0.2$, respectively. Hence, in the presence of a non-zero mean wall potential, the EO flow of a fluid of a smaller power-law index will be more sensitively affected by the phase shift between the wall patterns.

We next show in Fig. 4 how the flow rate may be affected by the wall undulation amplitude $\gamma$, where the phase shift $\phi=0$ for $\gamma<0$ is equivalent to $\phi=\pi$ for $\gamma>0$. First, when $\alpha=1$ and $\beta=0$ (i.e., uniform wall potential), the flow rate will decrease monotonically as the wall undulation increases in amplitude; see Fig. 4(a). This is expected since the resistance to flow increases as the narrowest section becomes narrower. The figure reveals that a shearthinning fluid is less susceptible than a shear-thickening fluid to this decreasing effect due to increasing $|\gamma|$ on the flow rate. Second, when $\alpha=0$ and $\beta=1$ (i.e., zero mean wall potential), the flow rate is an odd function of $\gamma$, and varies non-monotonically with $\gamma$; see Fig. 4(b). In the absence of wall undulation $(\gamma=0$, or flat walls), the net flow rate is always zero for any $n$. The non-monotonic variation of $\hat{q}$ with $\gamma$ arises from two competing effects. As $\gamma$ increases in magnitude, the area of the narrowest section decreases (thereby decreasing the flow as explained above), but on the other hand there is a stronger interaction between the wall undulation and the wall potential modulation (thereby enhancing the flow, whether positive or negative). The optimum value of $\gamma$, for which the flow rate is the maximum 
in magnitude, is larger for smaller $n$. Third, when $\alpha=1$ and $\beta=1$, the flow rate also varies non-monotonically with $\gamma$, but $\hat{q}-\left.\hat{q}\right|_{\gamma=0}$ is no longer an odd function of $\gamma$; see Fig. 4(c). Again, it is seen that the flow rate can increase more dramatically as $\gamma$ approaches the optimum value for smaller $n$. The ratio of $\hat{q}_{\max }$ to $\left.\hat{q}\right|_{\gamma=0}$ is equal to $1.07,1.13,1.34,2.92$ for $n=2,1,0.5,0.2$, respectively. This implies that, for a wall potential with non-zero mean and modulation components, the EO flow of a fluid of a smaller power-law index will be more sensitively affected by the amplitude of the wall undulation.

As has been remarked earlier, in the Newtonian case $(n=1)$, the wall potential modulation $\beta$ will have no effect on the flow rate when either $\gamma$ or $\cos \phi$ is zero. This statement can be extended to the non-Newtonian cases $(n \neq 1)$ only when $\alpha=0$. For $\alpha \neq 0$, this trait of independence of $\beta$ is no longer applicable to $n \neq 1$. As is shown in Fig. 5, where $\alpha=1$, the flow rate will increase, keep constant, or decrease as $\beta$ increases, depending on $n<1, n=1$, or $n>1$, respectively. These distinguishing behaviors are exhibited when either $\gamma=0$ (Fig. 5(a)) or $\cos \phi=0$ (Fig. 5(b)). Results shown in this figure are also in sharp contrast to what was found in a previous study [26]: with a Newtonian depletion layer, the wall potential modulation will not have any effect on the flow rate, for any $n$, when there is no applied pressure forcing. Here, it is seen that in the absence of a depletion layer, the wall potential modulation will have finite effect on the flow rate for $n \neq 1$ and $\alpha \neq 0$. One can readily reason that, for $n \neq 1$, a sinusoidal wall potential distribution of a mean $\alpha \neq 0$ will not generate a flow rate equal to that resulting from a uniform wall potential of $\alpha$. This is simply due to the nonlinear stress-shear rate relationship for $n \neq 1$. As $\beta$ increases, the wall stress increases in the region near the peak wall potential, which for a shear-thinning fluid $(n<1)$ means a decreased effective viscosity and hence leads to an overall increased flow. The opposite is true for a shear-thickening fluid $(n>1)$. One may make use of these distinguishing behaviors to determine, based on experimental observations, whether a fluid under investigation is shear-thinning, shear-thickening, or Newtonian.

Finally, we show in Fig. 6, for an example case, the stress distribution $\hat{\tau}(\hat{x}, \hat{y})$, and the corresponding flow pattern and pressure gradient $\hat{P}_{x}(\hat{x})$ in one periodic domain of the channel, where $n=0.5, \alpha=0.1, \beta=\gamma=0.5$, and $\phi=\pi$. In this example case, the stress type varies from Cases 2 through 5 over one period of the wall pattern. The shear stress has a sharp 
gradient in the near-wall EDL, where the electric forcing is effective. Outside the EDL, the stress has a linear cross-sectional profile, which is characteristic of a pressure-driven flow.

To maintain continuity of flow, the pressure gradient $\hat{P}_{x}$ is negative (i.e., adverse pressure gradient) near the region where the electric forcing is the positive maximum, but is positive (i.e, favorable pressure gradient) near the region where the electric forcing is the negative maximum. The negative wall potential results in a recirculation zone near the wall in the bulging part of the channel.

\section{Concluding remarks}

A numerical method has been developed to solve the problem of nearly one-dimensional electroosmotic flow of a power-law fluid through a slit microchannel with gradually varying channel height and wall potential. The method involves the searching for a flow rate that will give rise to an axial pressure distribution with zero pressure difference over one wavelength. For a general flow behavior index $n$, the trial-and-error solution seeking can be tedious and time-consuming. For $n$ equal to the reciprocal of an integer, the solution searching can be accomplished more easily as polynomial equations can be deduced to relate the flow rate and the local pressure gradient. When $n$ is the reciprocal of an even integer, we also need to distinguish between the various types of the stress distribution as controlled by the local pressure gradient, wall height and wall potential.

We have shown that, for a given non-zero wall potential modulation $(\beta)$, there exist an optimum value of the wall undulation amplitude $(\gamma)$ for which the flow rate is the maximum, where the flow rate is more sensitively affected by the mean wall potential $(\alpha)$ for smaller $n$. We have also shown that, when the mean wall potential is non-zero, the flow behavior index will have qualitatively different effects on how the wall potential modulation may interact with the wall undulation in driving the flow. For $n=1$ (i.e., Newtonian fluid), the wall potential modulation will have no effect on the flow rate when either the walls are flat $(\gamma=0)$ or the two wall patterns are at a phase shift of $\phi=\pi / 2$. This statement can be extended to $n \neq 1$ only if the mean wall potential is zero. Under a positive mean wall potential, increasing the amplitude of the wall potential modulation may increase or decrease the flow 
rate, depending on $n<1$ or $n>1$, respectively. Such a distinguishing behavior can be utilized to verify whether a fluid under investigation is shear-thinning or not. This finding has not been reported in the literature before.

\section{Acknowledgments}

The work was supported by the Research Grants Council of the Hong Kong Special Administrative Region, China, through Project No. HKU 715510E, and also by the University of Hong Kong through the Small Project Funding Scheme under Project Code 201309176109. 


\section{References}

[1] D. Burgreen, F.R. Nakache, Electrokinetic flow in ultrafine capillary slits, J. Phys. Chem. 68 (1964) 1084-1091.

[2] C. Zhao, C. Yang, Electrokinetics of non-Newtonian fluids: A review, Adv. Colloid Interface Sci. 201-202 (2013) 94-108.

[3] S. Das, S. Chakraborty, Analytical solutions for velocity, temperature and concentration distribution in electroosmotic microchannel flows of a non-Newtonian bio-fluid, Anal. Chim. Acta 559 (2006) 15-24.

[4] S. Chakraborty, Electroosmotically driven capillary transport of typical non-Newtonian biofluids in rectangular microchannels, Anal. Chim. Acta 605 (2007) 175-184.

[5] C.L.A. Berli, M.L. Olivares, Electrokinetic flow of non-Newtonian fluids in microchannels, J. Colloid Interface Sci. 320 (2008) 582-589.

[6] A.M. Afonso, M.A. Alves, F.T. Pinho, Analytical solution of mixed electroosmotic/pressure driven flows of viscoelastic fluids in microchannels, J. Non-Newtonian Fluid Mech. 159 (2009) 50-63.

[7] C.O. Ng, Combined pressure-driven and electroosmotic flow of Casson fluid through a slit microchannel, J. Non-Newtonian Fluid Mech. 198 (2013) 1-9.

[8] C.O. Ng, C. Qi, Electroosmotic flow of a viscoplastic material through a slit channel with walls of arbitrary zeta potential, Phys. Fluids 25 (2013) 103102.

[9] C. Zhao, E. Zholkovskij, J. Masliyah, C. Yang, Analysis of electroosmotic flow of powerlaw fluids in a slit microchannel, J. Colloid Interface Sci. 326 (2008) 503-510.

[10] M.L. Olivares, L. Vera-Candioti, C.L.A. Berli, The EOF of polymer solutions, Electrophoresis 30 (2009) 921-929.

[11] C. Zhao, C. Yang, Nonlinear Smoluchowski velocity for electroosmosis of power-law fluids over a surface with arbitrary zeta potentials, Electrophoresis 31 (2010) 973-979. 
[12] A. Babaie, A. Sadeghi, M.H. Saidi, Combined electroosmotically and pressure driven flow of power-law fluids in a slit microchannel, J. Non-Newtonian Fluid Mech. 166 (2011) 792-798.

[13] M.A. Vakili, A. Sadeghi, M.H. Saidi, A.A. Mozafari, Electrokinetically driven fluidic transport of power-law fluids in rectangular microchannels, Colloid Surf. A: Physicochem. Eng. Asp. 414 (2012) 440-456.

[14] J. Dhar, U. Ghosh, S. Chakraborty, Alterations in streaming potential in presence of time periodic pressure-driven flow of a power law fluid in narrow confinements with nonelectrostatic ion-ion interactions, Electrophoresis 35 (2014) 662-669.

[15] Q. Zhu, S. Deng, Y. Chen, Periodical pressure-driven electrokinetic flow of power-law fluids through a rectangular microchannel, J. Non-Newtonian Fluid Mech. 203 (2014) $38-50$.

[16] C. Zhao, C. Yang, An exact solution for electroosmosis of non-Newtonian fluids in microchannels, J. Non-Newtonian Fluid Mech. 166 (2011) 1076-1079.

[17] C. Zhao, C. Yang, Electroosmotic flows of non-Newtonian power-law fluids in a cylindrical microchannel, Electrophoresis 34 (2013) 662-667.

[18] J. L. Anderson, W.K. Idol, Electroosmosis through pores with nonuniformly charged walls, Chem. Eng. Commum. 38 (1985) 93-106.

[19] D. Long, H.A. Stone, A. Ajdari, Electroosmotic flows created by surface defects in capillary electrophoresis, J. Colloid Interf. Sci. 212 (1999) 338-349.

[20] A.E. Herr, J.I. Molho, J.G. Santiago, M.G. Mungal and T.W. Kenny, Electroosmotic capillary flow with nonuniform zeta potential, Anal. Chem. 72 (2000) 1053-1057.

[21] A.D. Stroock, M. Weck, D.T. Chiu, W.T.S. Huck, P.J.A. Kenis, Patterning electroosmotic flow with patterned surface charge, Phys. Rev. Lett. 84 (2000) 3314-3317.

[22] C.O. Ng, Q. Zhou, Electro-osmotic flow through a thin channel with gradually varying wall potential and hydrodynamic slippage, Fluid Dyn. Res. 44 (2012) 055507. 
[23] A. Ajdari, Electro-osmosis on inhomogeneously charged surfaces, Phys. Rev. Lett. 75 (1995) 755-758.

[24] A. Ajdari, Generation of transverse fluid currents and forces by an electric field: Electroosmosis on charge-modulated and undulated surfaces, Phys. Rev. E 53 (1996) 49965005.

[25] S. Ghosal, Lubrication theory for electro-osmotic flow in a microfluidic channel of slowly varying cross-section and wall charge, J. Fluid Mech. 459 (2002) 103-128.

[26] C.O. Ng, C. Qi, Electroosmotic flow of a power-law fluid in a non-uniform microchannel, J. Non-Newtonian Fluid Mech. 208-209 (2014) 118-125.

[27] C. Qi, C.O. Ng, Electroosmotic flow of a power-law fluid through an asymmetrical slit microchannel with gradually varying wall shape and wall potential, Colloid Surf. A: Physicochem. Eng. Asp. 472 (2015) 26-37.

[28] L.G. Leal, Advanced Transport Phenomena, Cambridge University Press, 2007.

[29] W.M. Deen, Analysis of Transport Phenomena, 2nd ed., Oxford University Press, 2012.

[30] M. Kiguchi, M. Nakayama, K. Fujiwara, K. Ueno, T. Shimada, K. Saiki, Accumulation and depletion layer thickness in organic field effect transistors, Jpn. J. Appl. Phys. 42 (2003) L1408.

[31] R.F. Probstein, Physicochemical Hydrodynamics, Butterworths, New York, 1989. 


\section{Figure captions}

Figure 1 Electroosmotic flow of a power-law fluid through a slit microchannel with undulating and non-uniformly charged walls, where $(x, y)$ are the axial and transverse coordinates. The wall shape $y=h(x)$ and the wall potential $\zeta=\zeta(x)$ are periodic functions of $x$ with a wavelength $L$, which is much longer than half the average channel height $h_{0}$.

Figure 2 Six possible cases of stress distributions $\hat{\tau}(\hat{y})$ for $0 \leq \hat{y} \leq \hat{h}(\hat{x})$, depending on the local values of pressure gradient $\hat{P}_{x}(\hat{x})$, channel height $\hat{h}(\hat{x})$, and wall potential $\hat{\zeta}(\hat{x})$. See Table 1 for the ranges of $\hat{\zeta}(\hat{x})$ and $\hat{P}_{x}(\hat{x})$ corresponding to these six cases.

Figure 3 Flow rate $\hat{q}$ as a function of the phase shift $\phi$, for (a) $\alpha=0, \beta=1, \gamma=0.5$; (b) $\alpha=1, \beta=1, \gamma=0.5$.

Figure 4 Flow rate $\hat{q}$ as a function of the amplitude of the wall undulation $\gamma$, for (a) $\alpha=1, \beta=0, \phi=0 ;$ (b) $\alpha=0, \beta=1, \phi=0 ;$ (c) $\alpha=1, \beta=1, \phi=0$.

Figure 5 Flow rate $\hat{q}$ as a function of the amplitude of the wall potential modulation $\beta$, for (a) $\alpha=1, \gamma=0$; (b) $\alpha=1, \gamma=0.5, \phi=\pi / 2$.

Figure 6 In one wavelength of the channel, $0 \leq \hat{x} \leq 1,0 \leq \hat{y} \leq \hat{h}(\hat{x})$, (a) stress distribution $\hat{\tau}(\hat{x}, \hat{y})$, which varies among different cases in different regions as indicated; (b) streamlines; (c) pressure gradient distribution $\hat{P}_{x}(\hat{x})$, for $n=0.5, \alpha=0.1, \beta=0.5, \gamma=0.5$, and $\phi=\pi$. 


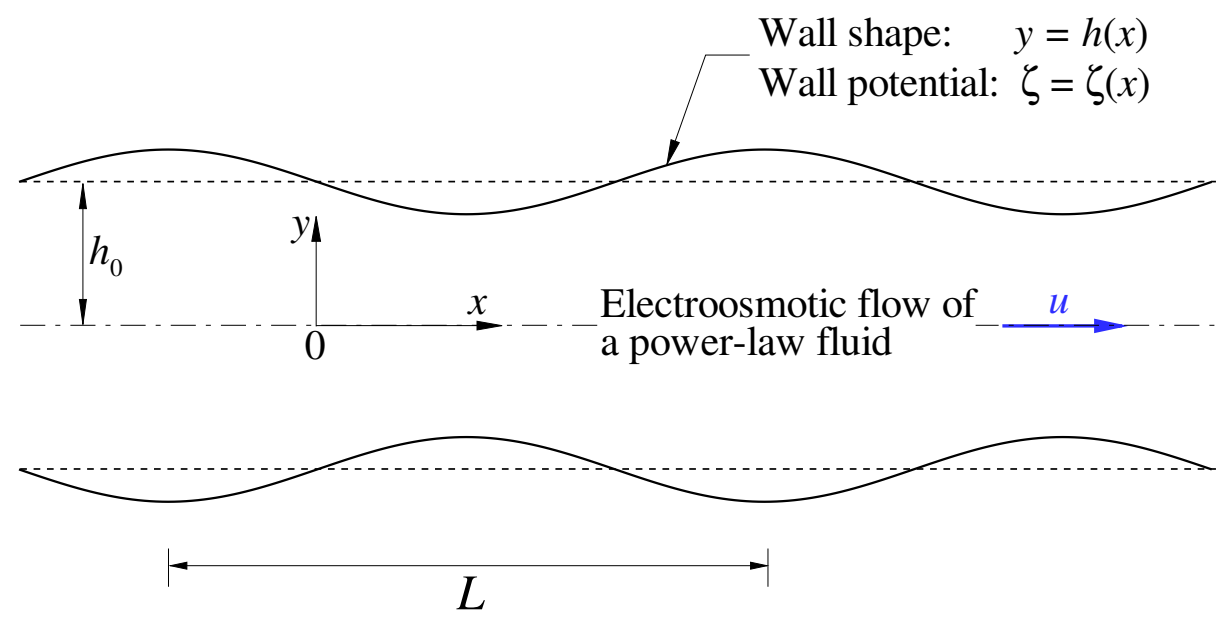

Figure 1: Electroosmotic flow of a power-law fluid through a slit microchannel with undulating and non-uniformly charged walls, where $(x, y)$ are the axial and transverse coordinates. The wall shape $y=h(x)$ and the wall potential $\zeta=\zeta(x)$ are periodic functions of $x$ with a wavelength $L$, which is much longer than half the average channel height $h_{0}$. 


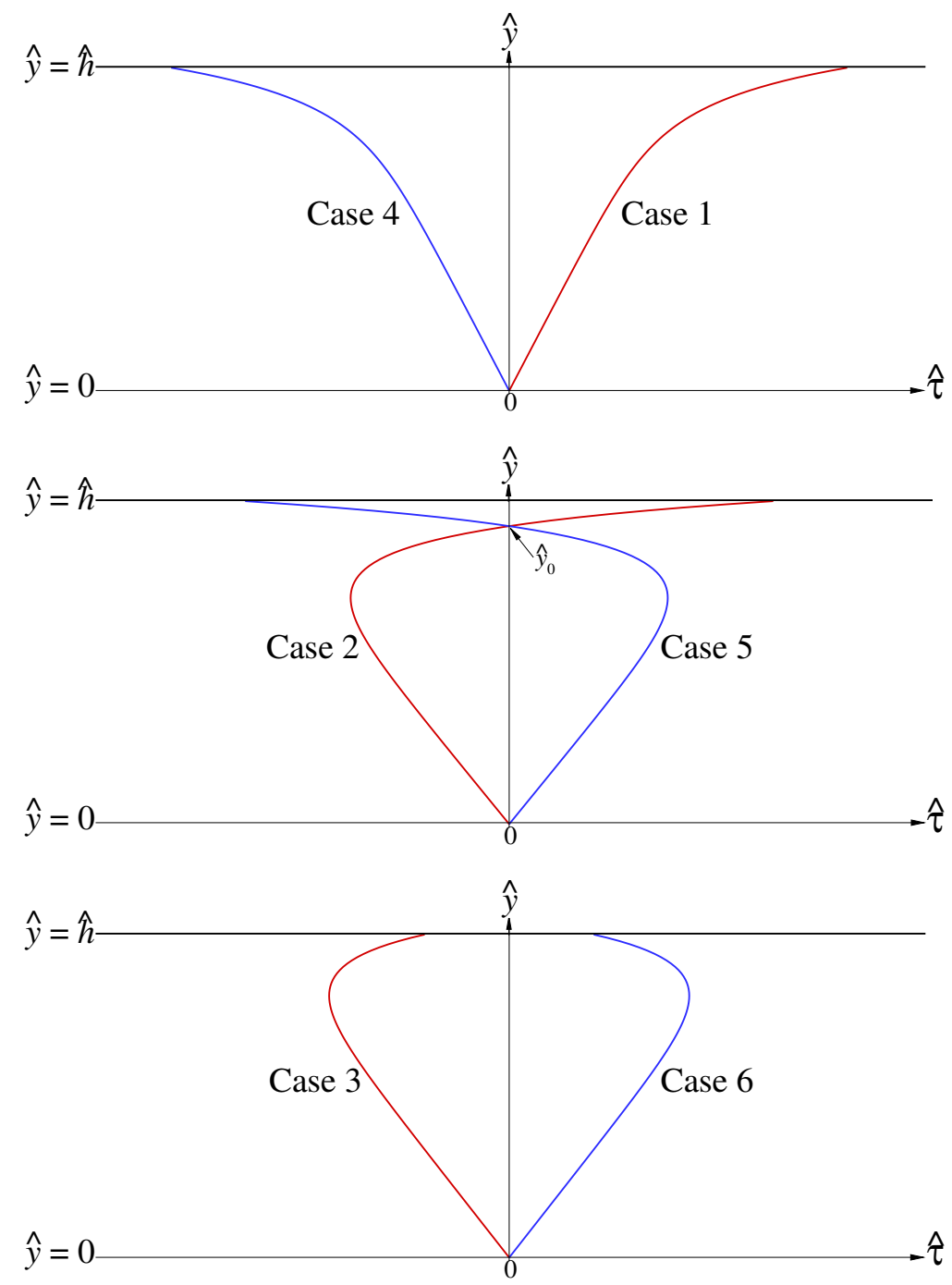

Figure 2: Six possible cases of stress distributions $\hat{\tau}(\hat{y})$ for $0 \leq \hat{y} \leq \hat{h}(\hat{x})$, depending on the local values of pressure gradient $\hat{P}_{x}(\hat{x})$, channel height $\hat{h}(\hat{x})$, and wall potential $\hat{\zeta}(\hat{x})$. See Table 1 for the ranges of $\hat{\zeta}(\hat{x})$ and $\hat{P}_{x}(\hat{x})$ corresponding to these six cases. 

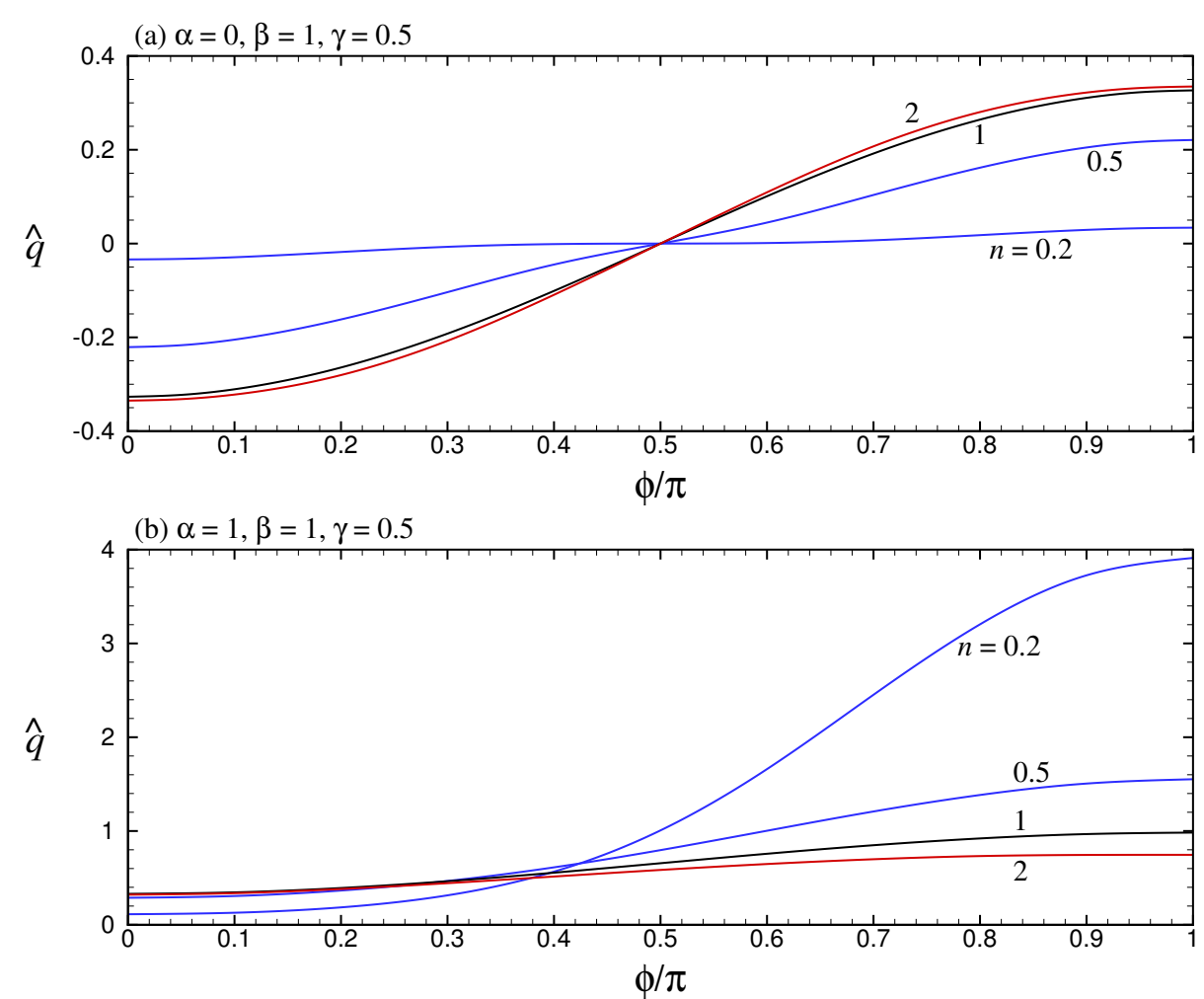

Figure 3: Flow rate $\hat{q}$ as a function of the phase shift $\phi$, for (a) $\alpha=0, \beta=1, \gamma=0.5$; (b) $\alpha=1, \beta=1, \gamma=0.5$. 

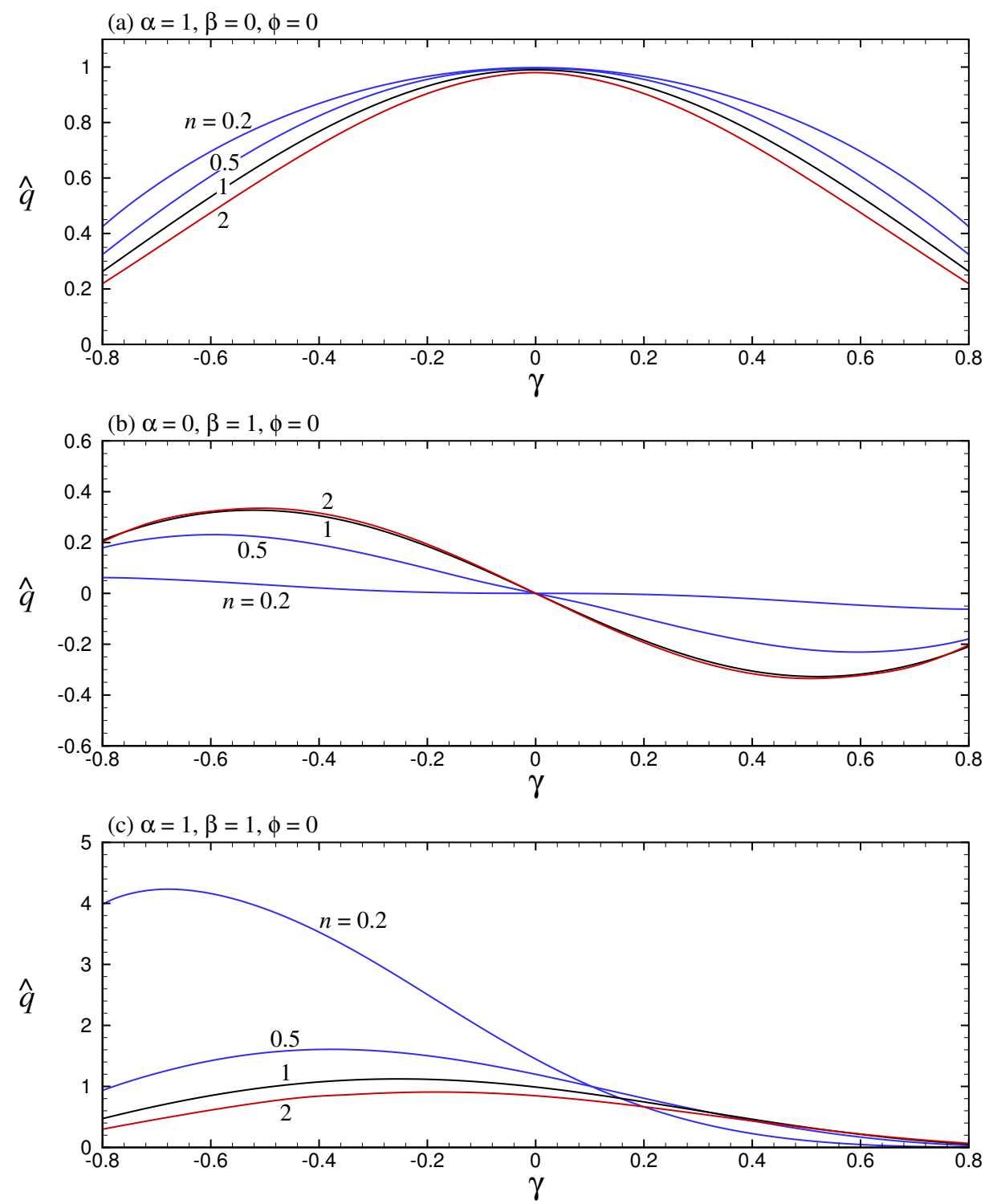

Figure 4: Flow rate $\hat{q}$ as a function of the amplitude of the wall undulation $\gamma$, for (a) $\alpha=1$, $\beta=0, \phi=0 ;$ (b) $\alpha=0, \beta=1, \phi=0 ;$ (c) $\alpha=1, \beta=1, \phi=0$. 

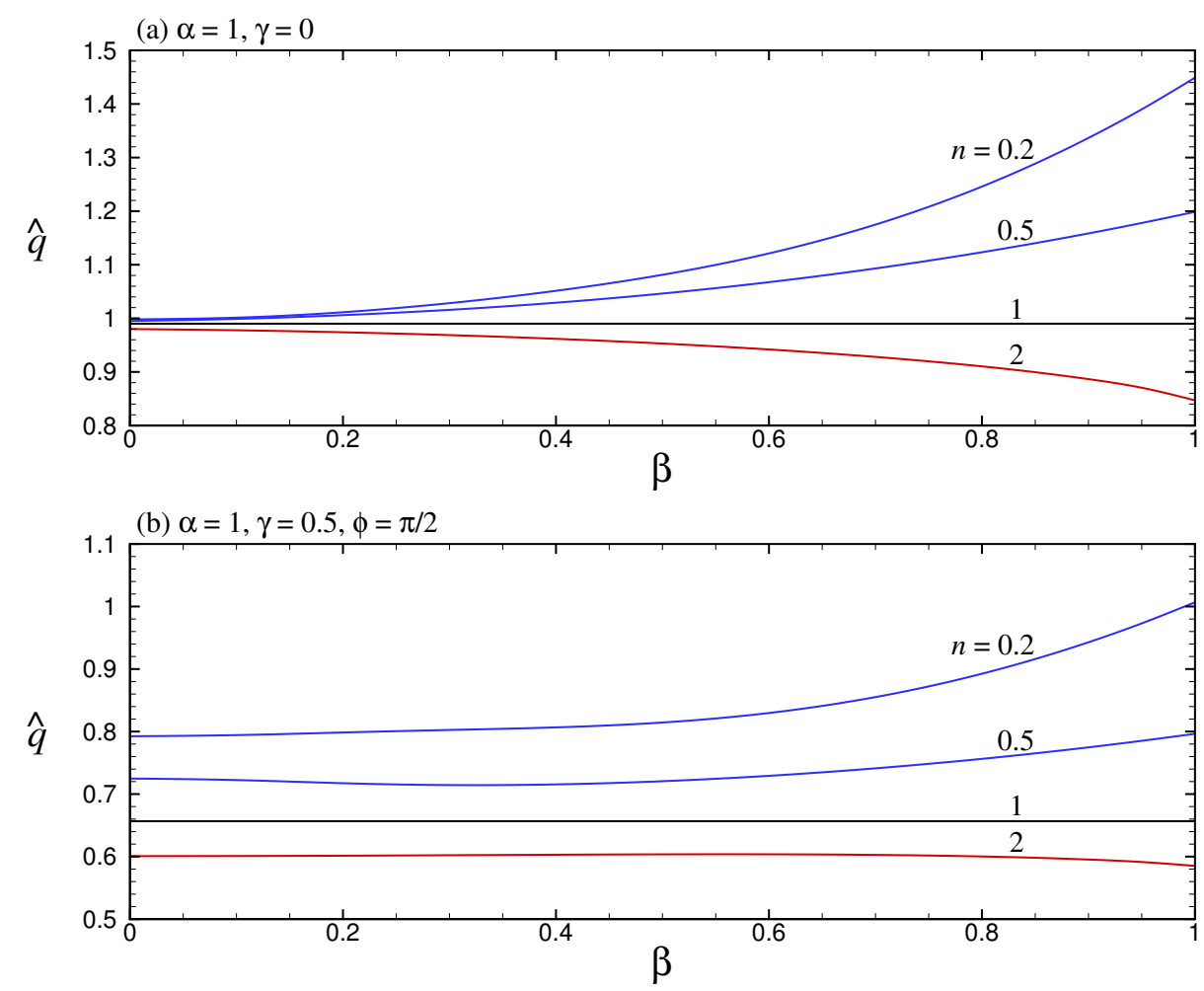

Figure 5: Flow rate $\hat{q}$ as a function of the amplitude of the wall potential modulation $\beta$, for (a) $\alpha=1, \gamma=0$; (b) $\alpha=1, \gamma=0.5, \phi=\pi / 2$. 
(a) Stress $\hat{\imath}(\hat{x}, \hat{y})$

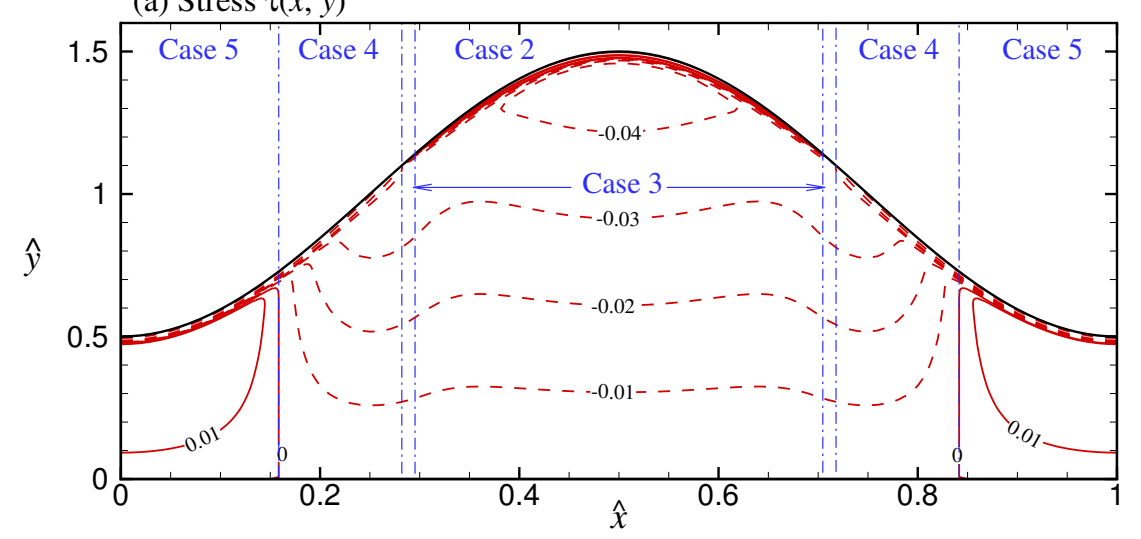

(b) Streamlines
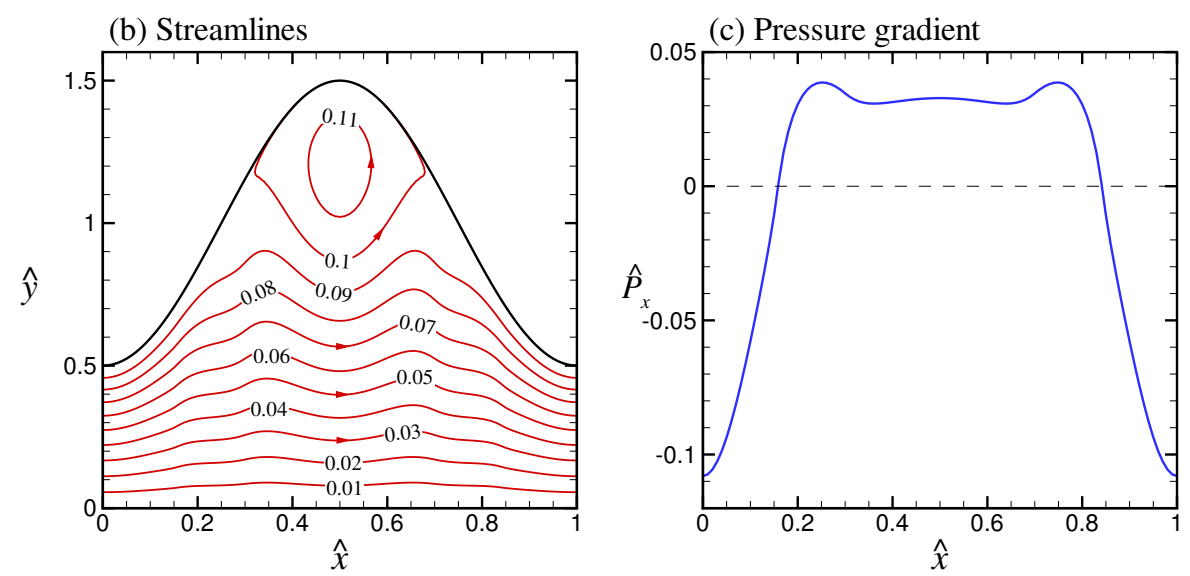

Figure 6: In one wavelength of the channel, $0 \leq \hat{x} \leq 1,0 \leq \hat{y} \leq \hat{h}(\hat{x})$, (a) stress distribution $\hat{\tau}(\hat{x}, \hat{y})$, which varies among different cases in different regions as indicated; (b) streamlines; (c) pressure gradient distribution $\hat{P}_{x}(\hat{x})$, for $n=0.5, \alpha=0.1, \beta=0.5, \gamma=0.5$, and $\phi=\pi$. 